\title{
A Dialogical Trial for Understanding a Mother and a Child with Autism Spectrum Disorder: Preliminary Implication for Nursing and Care
}

\section{Mami Yamamoto ${ }^{1,2 *}$, Midori Asano ${ }^{3}$ and Naoki Nomura ${ }^{4}$}

${ }^{1}$ Assistant professor, Department of Nursing of Children and Child Rearing Families at Gifu College of Nursing in Hashima City, Gifu, Japan ${ }^{2}$ Graduate School of Medicine, Nagoya University in Nagoya City, Aichi, Japan

${ }^{3}$ Professor in the Department of Nursing for Developmental Health at Graduate School of Medicine, Nagoya University in Nagoya City, Aichi, Japan

${ }^{4}$ Professor in the Department of Intercultural Studies at Graduate School of Humanities and Social Sciences in Nagoya City University in Nagoya City, Aichi, Japan

\begin{abstract}
The objective of this study is to demonstrate the change in qualitative levels of the context (evolution of context) in communication between a mother and a child with autism spectrum disorder (ASD).
\end{abstract}

In order to realize this objective, we focus on the relationship between contexts, and describe dynamic change inside mother-child communication. The data were collected thorough fieldwork by interview to the mother and participant observation. We analyzed about qualitative level of a context by qualitative analysis.

When the mother and the child with ASD experience repeated cycles of conflict and trial and error, the qualitative levels of the shared context of communication become comprehensive. The subject, which is the change in communication, is a context that arises in the parent and child's whole unit. Synchronously, the context about how to understand the communication between the parent and child becomes comprehensive. We propose that the dialogic partner's context and mother-child's context evolves in synchrony. When the object of nursing becomes a unit, including the nursing care provider, nursing can be asserted as a change of relationship in the interaction between the nursing care provider and the mother and child.

Keywords: Autism spectrum disorder; Narrative; Interaction; Mother-child; Child rearing; Gregory bateson; Qualitative research

Abbreviations: ASD: Autism Spectrum Disorder

\section{Introduction}

In this paper, we demonstrate the change in qualitative levels of the context (evolution of context) of communication between a mother and a child with suspected autism spectrum disorder (ASD).

Autism spectrum disorder (ASD) is known as a communication disorder. There is a shared feeling between the mother and the child in terms of the difficulties encountered in ASD [1,2]. Mothers of ASD children have difficulty understanding their child's feelings, and as they gradually understand the emotions of their child, the mother and child form a unique bond that leads to understanding [3-6]. Moreover, mothers acquire coping strategies for raising a child with ASD [7-12]. Many previous studies have reported the viewpoint of understanding the communication between the mother and the child with ASD. These include the attachment pattern of the child with ASD [13] the relationship between the mother and the child with ASD [3]; the experience of the mother raising an autistic child [12,14-16]; the life of the family of an autistic child $[17,18]$; experience of the family, which is a polyphonic narrative [19]; and how the context of autism shapes maternal meaning-making and subjectivity [20]. However, no study has attempted to understand the change in qualitative levels of the context in communication between a mother and a child with ASD. Moreover, no study has affirmed a nursing care practitioner's recognition of mother-child communication. In this paper, we focus on determining the qualitative levels of shared context between a mother and her child with ASD.

The paper is based on ideas regarding communication proposed by Bateson [20] who stated that one fundamental principle of communication is reciprocal relationships. Bateson focused on "contexts" that are rules that help decipher messages sent by others. "A 'bit' of information," he said, "is definable as a difference which makes a difference. Such a difference, as it travels and undergoes successive transformation in a circuit, is an elementary idea" [21]. In other words, he posited that ideas are not born within the individual, but rather between the units of a reciprocal relationship. When we communicate, for example, we receive the messages associated with words, expressions, and actions alongside the context that is born within the unit, and we interpret the messages based on this context. Bateson stated that "the ecology of ideas that constitutes a small subsystem is called a context" [21]. Context is not something that exists independently of action or utterance, but "utterance or action, as part of the ecological subsystem, is called a context" [21]. The context determines the meaning of messages, which can change depending on how the communication is divided. In this paper, based on Bateson's idea, we define communication as a process of mutual influence, and a view of circular causality that makes an interaction a basic principle. The meaning and relationship are accruing inside the unit of interaction. Additionally, we think that a dialogue is an interaction including not only the exchange of language or action but also the process of influencing each other. The objective of this study is to demonstrate the change in qualitative levels of the context (evolution of context) of communication between a mother and a child with ASD. The new viewpoints described in this paper will help nursing care providers understand the structures of meaningwhich are vertically layered rather than parallel-in parent-child communications. We also propose the recognition of dialogic partner for understand the mother-child communication.

*Corresponding author: Mami Yamamoto, Gifu College of Nursing, 3047-1 Egira, Hashima City, Gifu, 501-6295, Japan, Tel/Fax: +81-58-397-2340; E-mail myamamoto@gifu-cn.ac.jp

Received February 20, 2013; Accepted July 28, 2013; Published August 02 , 2013

Citation: Yamamoto M, Asano M, Nomura N (2013) A Dialogical Trial for Understanding a Mother and a Child with Autism Spectrum Disorder: Preliminary Implication for Nursing and Care. J Nurs Care 2: 130. doi:10.4172/21671168.1000130

Copyright: (c) 2013 Yamamoto M, et al. This is an open-access article distributed under the terms of the Creative Commons Attribution License, which permits unrestricted use, distribution, and reproduction in any medium, provided the original author and source are credited. 


\section{Methods}

\section{Study design}

This article is a single case study of a mother and a child with suspected ASD. Because our study required a detailed description of interaction and detailed consideration supported by theory, we relied on Bateson's "Logical Categories of Learning and Communication" [21] about the view that the qualitative level of a context. In this research, we took into account the relationship during interaction. Therefore, I describe the relationship by indicating scans that of a dialogue between the mother and the first author or communication between the mother, child, and first author. Moreover, the first author's subjectivity, reflected in an idea or a feeling, was described because the first author's consideration during interaction was essential in identifying recognition for dialogic nursing practice.

\section{Data collection}

Procedure: I (here in after 'I' signifies the first author) participated in the Sakura classroom as a volunteer staff member for three years starting in February 2009. In the Sakura classroom, parents and children who have difficulty communicating participated together. The purpose of this classroom was to support children's development and to provide parents with an approach for understanding their child's feelings through activity or discussions with fellow members. I recruited participants for this research from the Sakura classroom and the like, and seven children and their mothers participated. The child and mother who is picked up this article are one of these pairs.

Before beginning this research, I received approval from the Nagoya University School of Medicine Ethics Committee regarding the ethical considerations related to this research, and the benefits and disadvantages of participation in the research. Then, I explained the purpose, the methods, benefits and disadvantages, and the ethical considerations of this research using document to the administrator of the Sakura classroom; and received permission from the school. I also explained this research to the mother, showing her this document, and obtained her consent. The names of the participants and institutions have been changed to fictitious names.

Fieldwork: Data were collected through participant observation and interviews during fieldwork. We referred to the ethnography $[22,23]$ and narrative research $[24,25]$. The mother and her son's name is Aya and Kenji. I met Aya and Kenji once a week for approximately one year in the Sakura classroom and visited their home once every one to three months for two years. My field notes described the activities and the atmosphere of the classroom, exchanges of action and language between the mother and child, the atmosphere of the pair, and my general ideas regarding the classroom. During the interviews at their home, the mother freely talked about topics of her choosing, which were related to childrearing? I asked detailed questions using "the notknowing position" [24] after the mother expressed her feelings and ideas. While visiting their house, I established an agreement with the mother and recorded the dialogs with an IC recorder, and I created transcripts of these dialogs promptly after the visit. In addition, I drafted field notes that described my thoughts during the visit. I asked the mother about the burden of participating in this research periodically, and I informed her of her right to terminate participation in this study.

Moreover, I wrote down any changes in my ideas as well as questions that arose concerning the seven children and their mothers as part of my field notes during the fieldwork.

Our data consisted of field notes, recorded dialogs, and transcripts.

\section{Data analysis}

During my fieldwork, I sensed that Aya and Kenjis communication were difficult, as they often resorted to trial and error during their communication. Focusing on this communication, I analyzed its level of context. Aya and Kenji's inability to communication feelings was what they called 'BangBang.' The BangBang was the main theme of the dialog for two years, Aya and me. Depend on method of qualitative analysis [26], narrative and scene about BangBang was grouped in the unit of the meaning. And I arranged the qualitative level of the context in BangBang as a logical type. The feeling of the child was interpreted by both the mother's and my idea. In the analysis, we collated the changes in the qualitative levels of context as a vertical layered structure, based on the context that the mother and the child have already shared.

For the analysis about the dialog of this pair and the first author, I engaged in discussions with graduate students and researchers in the nursing domain, which the second author supervised. In addition, I solicited advice from the mother and revised it, and with the second author, I discussed my cognition regarding dialog with the mother and child. I consulted with the third author as well as graduate students of intercultural studies about understanding of the theory, and the third author advised about the arrangement of the level of context.

\section{Results and Conclusion}

\section{Introduction of Aya and Kenji}

Aya was in her thirties and Kenji was 31 months old when I met them. A serious communication was BangBang for Aya and Kenji. BangBang is a behavior in which he knocks his head vigorously against a floor or a table, which he began exhibiting about ten months after his birth. Aya thought BangBang was an action Kenji did when he gets tired of everything. Because Kenji did BangBang in places where he could be injured, such as on asphalt and in conspicuous places like a supermarket, Aya was anxious about when and where his BangBang would start and whether she would be able to leave a particular place. BangBang was a strange action that she had not seen before. Therefore, BangBang had become a serious problem for Aya in raising Kenji. I spent time with Kenji and his elder sister every day, particularly during playtime.

\section{The logical type of context}

As described below, the context of BangBang shared between Aya and Kenji was evolving. The terms in parenthesis are the expressions and actions of Aya and Kenji, and are supplied to help the reader understand the meaning of the dialog. And '...' indicate an ellipsis.

The context of BangBang has been shared by Aya and Kenji: In this level, their context was a set of action and response. BangBang was the means by which Kenji expressed displeasure, and BangBang was an action response to Kenjis favorite things, for example, the sound of a vacuum cleaner for Aya. In this context, the sign and reaction was decided. However, BangBang did not necessarily stop through fixed means; the context of an action with a means was not always shared between Aya and Kenji. Moreover, Kenji's BangBang action was a source of anxiety for Aya, who felt that the behaviour made him different from other children. Even if she could stop the behaviour of BangBang, her anxiety did not disappear. And Aya and Kenji started trial and error of search for meta-context about a set of action and response.

The context of BangBang is 'Kenji's personality': In the trial and error, Aya had Kenji examined by a psychologist, and in this way, Aya and Kenji got to context; the cautious personality such as to hard to be 
adapted to the slightest change in everyday living. This is a meta-context about the context of action and response, because it is the reason for the context of action and response.

However, this context gave rise to a conflict in another context. Following is Aya's narration.

Aya: I know it is just a personality trait because every child is the same. But maybe I want to feel relieved of this problem by thinking that he has a cautious personality or that he is sick. I don't want to say things like this, though. It has a deep meaning. (She spoke haltingly, searching for the right words.)

Aya: Maybe Kenji is different from others. I don't know his thoughts. But for Kenji, he thinks, this is who I am." Hmmm, but what if he thinks wrongly. (Suddenly a thought came to her, and she expressed it.) Actually everyone is similar, I mean five years old is five and six years old is six. Oh well, I guess it never ends! I don't know what is normal or not.

Aya had a question about the framework itself, which is cautious personality, that arose when she thought about "what is the meaning of normal?" and she began to consider how to know her own BangBang.

When I looked at BangBang for the first time, I tried to understand his action based on self-injurious behavior, which is an already acquired knowledge. However, I began to think that BangBang was a unique method of communication between mother and child when Kenji stopped the behavior as soon as Aya told him to. In this way, my own level of context toward understanding the mother-child communication deepened from base level knowledge to the meaning of mother and child.

The context of BangBang is 'relationship': In their 'Sorry-OK communication', the context that they shared was the relationship. Following is a scene of their Sorry-OK communication, which lasted $11 \mathrm{~min}$ and $34 \mathrm{~s}$.

One day when I visited, Aya said, "Wash your hands" to Kenji, who had returned home from kindergarten. Then, Kenji began to cry, saying "No!" Aya said to Kenji, "Let's go wash our hands together," but Kenji did not respond to this, and he began to shout "No, I won't!" A short time later, Kenji lay face down on the floor, kicking and struggling, and cried more loudly. Then, he placed both hands on his forehead and began to bang his head against the floor. Aya watched his actions, and instantly said in a strict tone, "Kenji! Don't BangBang!" Suddenly, Kenji stopped BangBang. Aya walked over to Kenji and picked him up, and he clung to her. Aya spoke to Kenji slowly:

Aya: Listen, Kenji. Please listen. Kenji, I’m so sorry. Sorry (she said gently again).

Kenji: (He whispered something, but we could not hear it.)

Aya: I'm sorry, Kenji.

Kenji: (He gradually settled down. A little later he said it again.) OK.

Aya: Did you say OK?

Kenji: Yes (speaking in small voice).

Aya: I'm sorry.

Kenji: I said OK, mom.

Aya: Well then, let's go wash your hands together.

Kenji: Got it.
Aya: OK. Let's go wash your hands.

Kenji: Just once though.

Aya: All right. What did you want to do when you came home?

Kenji: I am hungry! (Cheerful)

Aya: Oh, you are! Kenji, be sure to wash your hands when you come home, even if you are hungry, or you are sleepy. Please keep it in mind. Do you understand?

\section{Kenji: (He was not crying any longer.)}

Aya's narration about the Sorry-OK communication is as follows:

Aya: I was thinking about why Kenji does "BangBang." For now, maybe I want Kenji to know that I want to communicate with him, speak to him, or do things with him. ... I felt that Kenji seems to understand or relax when I listen to his words. When Kenji was crying, I asked him the reason why. "Because I wanted to use it," he began to say "I'm so sorry, Kenji," I apologized to him. Then he said, "It's OK, though." Through this incident, I thought about the importance of simple conversation between us. . . That I should make conversation with him for now. . . . Maybe I am becoming comfortable when we put our thoughts into words with each other. ... The thing is, it is a similar feeling to when a mother talks to a baby-the baby can understand even if it is in her womb. I wonder if he understands in this way.

In the Sorry-OK communication, the details for Kenji's disagreeable situation and the reason for Aya's apology were not expressed. This communication highlights the significance of the relationship emerging from their communication, which is not expressed by words. The shared context between the two of them is the relation which tries to understand each other. The shared context between Aya and Kenji deepened their relationship as they tried to understand each other. The context of this relationship is the meta-context that determines the context of Kenji's personality. This is because for the mother and the child, the question of whether Kenji's personality is a problem or not is determined by the type of relationship that Kenji and Aya desire to have with each other. In the context of the shared relationship based on SorryOK communication, BangBang becomes a form of communication in which Kenji's personality ceases to be a problem, and the mother and the child seek mutual understanding.

After witnessing the Sorry-OK communication, I realized that the relationship that emerges from this communication is more important for the mother and child, compared to stopping the BangBang action. I understood the importance of continuing to search for the meaning of the BangBang action with the mother and child.

The context of BangBang is changing beyond the context levels: Their context for the BangBang deepened from relationships to change and then comprehensively for Aya during our dialog. The authors analyzed continuously about the context of the communication between Aya and Kenji. One day when I visited Aya, I told her about our interpretation and asked for her thoughts, reaction, and sense of the interpretation, and whether it was incongruous. The next day, I received an e-mail from Aya. Following is the dialog between Aya and me in e-mail;

Aya: (About how Kenji cries every time he has to go to kindergarten). My problem disappears temporarily if I recognize that Kenji has special characteristics or some kind of disease (for example, he has anxiety about changes in his schedule) ... if Kenji is a child with such a character, as the psychologist says, then understanding him may also save him, which he can't convey himself. However, I might go back to the starting point in that way of thinking and attitude, but I have to. 
.. (Aya read our interpretation) I could take an objective view of myself and think that everything is meaningful. ...

Mami: Thank you for telling me your idea politely. I understand that your problem disappeared when you realized that it is a characteristic of Kenji, and by thinking this, you believe that the others understand Kenji's idea.... I am glad to hear that "everything is meaningful."

Aya: Yes, everything has a meaning, and it is all connected. His present way of communicating may also change into a different type as he grows up. I would like to get along with him slowly, when I have the time.

The context of Aya and Kenji evolved beyond the context levels through mutually understanding relations. She said that everything about BangBang has a meaning, and that it is all connected. She found meaning in the continuous relocation of the qualitative levels of the BangBang context. Their meaning of BangBang was told as the continuous change unity. Her flexibility such as 'I would like to get along with him slowly, when I have the time' appeared as feeling to the continuous loops which changes qualitative levels of the context.

The dialogs between the mother and child were a process for me to continuously rethink that what is the best way to understand their meaning. My own qualitative level of context for understanding the mother-child communication shifted from an already acquired knowledge to a unique relationship between mother and child, and finally the flexibility to change my awareness.

\section{Discussion}

\section{The communication between the mother and the child with ASD from the viewpoint of qualitative levels of context}

In this paper, we stated the change in qualitative levels of the context (evolution of context) of communication between the mother and the child suspected with ASD. The shared context level between mother and child eventually becomes more comprehensive and abstract, leading to shared contexts of contexts. Bateson [20] discussed the qualitative level between contexts in "Logical Categories of Learning and Communication" [27,28]. Our proposals that the mother-child context evolves into action and reaction, and that personality, relationship, and change become more comprehensive were supported by Bateson's theory. In the evolution of a context, parent-child trial and error arises. The mother and the child with suspected ASD repeat evolution cycles of shared context levels by trial and error. The previous studies reported mothers' flexibility: "mothers exhibit remarkable flexibility in the ability to adapt their lifestyles to meet their children's need" [9], Mothers experienced journey towards adaptation such as reacted to the stressor of an autism diagnosis, coped, and adapted over time" [29],"the mothers reported various strategies they used to manage their roles, their emotins, and their child's behaviors" [12].

Instead, we argue that such mother's flexibility is shifted toward the qualitative level of mother-child context. We likewise think that flexibility arises as a context evolves by trial and error. The dialogs between mother and child or between the nursing care provider and the mother/child might have worked to deepen the qualitative levels of contexts, because such relational exchanges would served to observe the changes of the context level, by looking at the context types from the outside. By sharing the process of moving the context level, mother and child could have sensed that they understood each other's feelings. A mother's sense of fulfillment toward understanding her child's feelings altered the mother-child relationship and worked to eliminate problems associated with parent-child relationships.

\section{Evolution of context}

The mother and the child with ASD are changing. The subject, which is the change in communication, is a context that is not one of the mother and the child, and arises in the parent and child's whole unit. The mother-child context is also evolving. In this paper, we described the change in the interaction by describing the first author's subjectivity in a dialog with the parent and child. When the parentchild communication changes, the context of the first author, who understands such communication, becomes more comprehensive.

We propose that the dialogic partner's context and motherchild's context evolves in synchrony. "The subjective and dialogical epistemology theory is useful for nursing practice" [30]. The recognition that this paper proposed induces a collaborative nursing view that involves the nursing care provider.

\section{A greater degree of freedom in writing about nursing}

In recognizing that the mother-child context and the nursing care provider context evolve in synchrony, the object of nursing, including the nursing care provider, becomes a whole unit. When the object of nursing becomes a unit, the new viewpoint of writing about nursing arises. In writing, nursing can be asserted as a change in the whole interaction between the nursing care provider and the mother and child. Sato [30] argued that the knowledge, which can be known at the inner side of an interaction, existed. It is the knowledge for telling the change in an interaction sensuously. Describing nursing as change inside interaction may convey knowledge as new quality.

\section{Limitations of This Study}

The work of this paper was to demonstrate the change in qualitative levels of the context in communication between mother and child with ASD. We demonstrate that evolution of context. Therefore, the contents of context this particular mother-child's will not be the same as another parent/child combination. We mentioned this as a part of the viewpoints for understanding mother-child communication.

Because the focus of this study was on both the infantile child and mother, the first author was unable to accurately determine the child's feelings. Although we made every effort to validate their observations by listening to the observations made by the mother regarding her child's feelings and her reasons, as well as spending long hours with the mother and child, we must admit the limitation that exists in the interpretation of the child's true feelings. Still, we believe that this work was valuable in understanding the shared contexts between a parent and child.

\section{Acknowledgement}

We thank our research participants for their cooperation. This research was provided by the grant of Japan Society of Nursing and Health Care. This paper is a part of a doctoral dissertation.

\section{References}

1. Miyamoto S (2008) The cause and pathophysiology of Developmental Disorder. In: Igarashi T, Hiraiwa M (Eds) The compass for clinical Pediatrics 2, an understanding and treatment of developmental disorder. NakayamaShoten Co Ltd. Tokyo, Japan.

2. Sheehan M (2010) Autism spectrum disorder. In: Allen PJ, Vessey JA, Schapiro $\mathrm{N}$ (Eds) Primary Care of the Child with a Chronic Condition. (5th Edn), Mosby Inc, St. Louis.

3. Kobayashi R (1996) Therapeutic intervention for affective communication with autistic infants in terms of relationship disturbances. Japanese Journal of Child and Adolescent Psychiatry 37: 319-330. 
Citation: Yamamoto M, Asano M, Nomura N (2013) A Dialogical Trial for Understanding a Mother and a Child with Autism Spectrum Disorder: Preliminary Implication for Nursing and Care. J Nurs Care 2: 130. doi:10.4172/2167-1168.1000130

4. Yamazaki N (2009) How does mother of autistic child "understand" her child? - Seeking the way towards autistic child from dialogue. Japanese Journal of Applied Psychology 34: 182-192.

5. Midence K, O'neill M (1999) The experience of parents in the diagnosis of autism. A pilot study. Autism 3: 273-285.

6. Yamamoto M, Kadoma A, kato M (2010) Experiential process of Mothers who Raise Children with Pervasive Developmental Disprders Including Autism. Journal of Japan Society of Nursing Research 33: 21-30.

7. Gray ED (2002) Ten years on: A longitudinal study of families of children with autism, Journal of Intellectual \& Developmental Disability, 27: 215-222.

8. Al Kandari MT (2006) Parenting and autistic child in Kuwait: Kuwaiti mothers' voice and experience with children labeled autistic. Syracuse University, Syracuse, New York.

9. Krausz M, Meszaros J (2005) The retrospective experiences a mother of a child with autism. The International Journal of Special Education 20: 36-46.

10. Marshall V, Long BC (2010) Coping processes as revealed in the stories of mothers of children with autism. Qual Health Res 20: 105-116.

11. Safe A, Joosten A, Molineux M (2012) The experiences of mothers of children with autism: managing multiple roles. J Intellect Dev Disabil 37: 294-302.

12. Rutgers $\mathrm{AH}$, Bakermans-Kranenburg $\mathrm{MJ}$, van ljzendoorn $\mathrm{MH}$, van BerckelaerOnnes IA (2004) Autism and attachment: a meta-analytic review. J Child Psychol Psychiatry 45: 1123-1134.

13. Markoulakis R, Fletcher P, Bryden $P$ (2012) Seeing the glass half full: benefits to the lived experiences of female primary caregivers of children with autism. Clin Nurse Spec 26: 48-56

14. Bilgin $H$, Kucuk $L$ (2010) Raising an autistic child: perspectives from Turkish mothers. J Child Adolesc Psychiatr Nurs 23: 92-99.

15. Larson E (2006) Caregiving and autism: How does children's propensity for routinization influence participation in family activities? Occupation Participation and Health 26: 69-79.

16. Marquenie K, Rodger S, Mangohig K, Cronin A (2011) Dinnertime and bedtime routines and rituals in families with a young child with an autism spectrum disorder. Aust Occup Ther J 58: 145-154.
17. Heslip CV (2010) Hispanic mothers' experiences of raising children with moderate to severe autism: A phenomenological study. Dissertation Abstract International: Section B: The Sciences and Engineering 70: 2899.

18. Mouzourou C, Santos RM, Gaffiney JS (2011) At home with disability: One family's three generations narrate autism. International Journal of Qualitative Studies in Education 24: 693-715.

19. Bateman JK (2011) Living Iminality: Maternal subjectivity in the context of raising children with autism. Publicly accessible Penn Dissertations. Paper 331

20. Bateson G (1972) Steps to an Ecology of Mind: Collected Essays in Anthropology, Psychiatry, Evolution, and Epistemology. The University of Chicago Press. London.

21. Roper MJ, Shapira J (2000) Ethnography in Nursing Research: Volume 1 of Methods in Nursing Research. Sage, London.

22. Kujiraoka T (2005) Preparing and delivering good case descriptions: For researchers and participant observers. University of Tokyo press. Tokyo.

23. Anderson H, Goolishian H (1992) The client is the expert: a Not-Knowing approach to Therapy. In: McNamee S, Kenneth GJ (Eds), Therapy as Social Construction. SAGE, London.

24. Sakurai A (2002) Interview as Sociology -How to hear a life story. Serikashobo, Tokyo.

25. Flick U (2009) An Introduction to Qualitative Research. (4th edn), SAGA London.

26. Nomura N (2008) Introduction to Bateson: What is communication? Kingo, Tokyo.

27. Nomura N (2012) Bateson for all: Let's Talk about learning! Kingo, Tokyo.

28. Lutz HR (2008) Coping with autism during childhood and adulthood: Mothers' journeys towards adaptation. Widener University School of Nursing, PA, USA.

29. Bowers R, Moore KN (1997) Bakhtin, nursing narratives, and dialogica consciousness. ANS Adv Nurs Sci 19: 70-77.

30. Sato Y (1984) Practicing Bateson. Contemporary philosophy 12: 140-149. 